\title{
Inflammatory signals are sufficient to elicit TOX expression in mouse and human CD8 ${ }^{+} T$ cells
}

\author{
Nicholas J. Maurice, ${ }^{1,2}$ Jacqueline Berner, ${ }^{3}$ Alexis K. Taber, ${ }^{1}$ Dietmar Zehn, ${ }^{3}$ and Martin Prlic ${ }^{1,4}$ \\ 'Vaccine and Infectious Disease Division, Fred Hutchinson Cancer Research Center, Seattle, Washington, USA. ${ }^{2}$ Molecular \\ and Cellular Biology Graduate Program, University of Washington, Seattle, Washington, USA. ${ }^{3}$ Division of Animal \\ Physiology and Immunology, Technical University of Munich School of Life Sciences Weihenstephan, Technical University \\ of Munich, Freising, Germany. ${ }^{4}$ Department of Immunology, University of Washington, Seattle, Washington, USA.
}

T cell receptor (TCR) stimulation leads to the expression of the transcription factor thymocyte selection-associated high-mobility group box (TOX). Prolonged TCR signaling, such as encountered during chronic infections or in tumors, leads to sustained TOX expression, which is required for the induction of a state of exhaustion or dysfunction. Although $\mathrm{CD} 8^{+}$memory T (Tmem) cells in mice typically do not express TOX at steady state, some human Tmem cells express TOX but appear fully functional. This seeming discrepancy between mouse and human $\mathrm{T}$ cells has led to the speculation that TOX is differentially regulated between these species, which could complicate the interpretation of preclinical mouse model studies. We report here that, similar to TCR-mediated signals, inflammatory cytokines are also sufficient to increase TOX expression in human and mouse Tmem cells. Thus, TOX expression is controlled by the environment, which provides an explanation for the different TOX expression patterns encountered in T cells isolated from specific pathogenfree laboratory mice versus humans. Finally, we report that TOX is not necessary for cytokinedriven expression of programmed cell death 1. Overall, our data highlight that the mechanisms regulating TOX expression are conserved across species and indicate that TOX expression reflects a T cell's activation state and does not necessarily correlate with $T$ cell dysfunction.

Authorship note: NJM and JB contributed equally to this work.

Conflict of interest: The authors have declared that no conflict of interest exists.

Copyright: ( ) 2021, Maurice et al. This is an open access article published under the terms of the Creative Commons Attribution 4.0 International License.

Submitted: April 21, 2021

Accepted: May 21, 2021

Published: July 8, 2021

Reference information: /CI Insight. 2021;6(13):e150744.

https://doi.org/10.1172/jci. insight.150744.

\section{Introduction}

$\mathrm{T}$ cell exhaustion (i.e., dysfunction) is driven by chronic $\mathrm{T}$ cell receptor (TCR) stimulation with cognate antigen (Ag; refs. 1-3). It describes a differentiation state in which T cells have diminished capacity to respond to stimulatory inputs and limited effector capacity (2-4). The purpose of $\mathrm{T}$ cell exhaustion during chronic infections may be to limit tissue pathologies when pathogen cannot be immunologically eliminated $(5,6)$. Though exhaustion could be considered an immunologic concession during chronic infection, it also occurs in tumors and causes an attenuated antitumor cytotoxic $\mathrm{T}$ cell response (7). Thus, mechanistically understanding and therapeutically overcoming $\mathrm{T}$ cell exhaustion has been a major goal of tumor immunotherapy. Chronic TCR stimulation elicits a program that leads to constitutively high expression of programmed cell death 1 (PD-1; ref. 8). PD-1 is an inhibitory receptor that is expressed by activated and exhausted $\mathrm{T}$ cells and is often used as a biomarker to infer T cell functionality (9). When bound to its ligands, PD-1 negatively regulates T cell function (2). Therapeutic targeting of PD-1 with monoclonal antibodies, also referred to as immune checkpoint inhibitors, can reinvigorate a subset of these PD-1-expressing T cells (2, 10-12).

A set of recent studies demonstrated that the transcription factor thymocyte selection-associated high-mobility group box (TOX) protein drives or stabilizes this TCR-mediated T cell dysfunction and PD-1 upregulation (6, 13-16). When stably expressed, TOX drives Ag-specific T cell exhaustion in mouse models of chronic lymphocytic choriomeningitis virus (LCMV) infection, transplantable B16 melanoma, and inducible hepatocellular carcinoma $(6,13,14)$. Further, putative tumor $\mathrm{Ag}$-specific $\mathrm{CD} 8^{+} \mathrm{T}$ cells isolated from primary human breast, ovarian, and skin cancer samples, as well as those specific for hepatitis $\mathrm{C}$ virus (HCV), mirror this phenotype, suggesting TOX dictates exhaustion programs in humans, too $(6,13,14)$. Of note, TOX expression by HCV-specific T cells is reduced after treatment and clearance of the infection, but it is still detectable at higher levels than in T cells from HCV infections that spontaneously resolve and among $\mathrm{T}$ cells specific for influenza A virus (IAV; ref. 6). Mechanistic insight is provided by targeted deletion of TOX in Ag-specific 
cytotoxic T cells, which diminishes PD-1 expression and restores functionality at the expense of cell survival (6, 13). Therefore, TOX concedes activation and effector function for exhaustion (i.e., PD-1 expression) and T cell survival during chronic TCR stimulation. In instances of brief TCR engagement, TOX is transiently induced to a level lower than that of exhausted $T$ cells, but with limited known functional consequence $(6,13,14)$.

Although the requirement for TOX has been well defined in the context of TCR-mediated dysfunction, there is nascent evidence that TOX expression by itself is not indicative of T cell exhaustion. Recent studies illustrated that TOX expression is detected in some functional $\mathrm{CD} 8^{+}$memory $\mathrm{T}$ (Tmem) cells, for instance, in $\mathrm{CD}^{+}$effector memory (Tem) and CD45RA-expressing Tem (Temra) subsets (17). CD8 ${ }^{+}$Tmem cells specific for the latent viruses, CMV and EBV, had elevated TOX expression, compared with those specific for acute infections, which further suggests that TCR signals are critical in regulating TOX expression (17). In a second study, it was shown that a fraction of the human Tmem population expresses TOX transcripts among other signature genes typically associated with T cell exhaustion (18). The observation that functional human Tmem cells express TOX also led to questions of whether TOX is functionally conserved between mouse and human T cells (19). Further complicating TOX and exhaustion, the murine tissue-resident Tmem (Trm) cell transcriptome is characterized by the concomitant expression of transcripts encoding Tox, exhaustion markers, TCR signaling components, and cytotoxic molecules, well after initial priming events $(20,21)$. Although the role of TOX in these TOX-expressing populations with and without signs of T cell exhaustion is not fully understood, these data suggest that TOX expression by Tmem cells cannot be reliably used to extrapolate T cell function.

Although the role of TCR signals in initiating and maintaining PD-1 and TOX expression has been well established, relatively little remains known about non-TCR signals that could regulate their expression in T cells (22). We considered that cytokine-mediated stimuli could also affect TOX expression levels without promoting the induction of T cell exhaustion. First, proinflammatory cytokines, such as IL-15, can induce PD-1 without agonist TCR signals (22). Second, Trm cells that are likely not detecting cognate Ag still upregulate PD-1 and other markers associated with exhaustion $(20,21,23,24)$, yet rely on IL-15 signaling for maintenance in some tissues $(25,26)$. Thus, inflammatory signals could provide an explanation for some of the seemingly disparate results of TOX expression and T cell function. Here, we show that proinflammatory cytokines were sufficient to induce TOX expression in the absence of agonist TCR signals in both mouse and human $\mathrm{CD}^{+} \mathrm{Tmem}$ cells while concurrently inducing the expression of cytotoxic molecules. Together, these data demonstrate that TOX expression per se does not indicate TCR-mediated dysfunction or even a recent TCR signals. We also demonstrate that PD-1 expression was still upregulated in TOX-deficient T cells, indicating that TOX was not necessary for PD-1 expression. Overall, our data reveal that TCR-independent mechanisms shape TOX and PD-1 expression heterogeneity in Tmem cells and indicate that they are conserved in both mouse and human $\mathrm{T}$ cells. Though these findings ultimately complicate the use of TOX exclusively as an exhaustion biomarker, they implicate TOX in inflammation-driven programs of Tmem cell activation.

\section{Results}

Cytokine stimulation induced TOX expression in murine CD8+ Tmem cells. The proinflammatory cytokines IL-12, IL-15, and IL-18 elicit IFN- $\gamma$ and granzyme B (GzmB) expression in mouse and human CD8 ${ }^{+}$Tmem cells in the absence of agonist TCR signals (27-29). We first sought to determine if these cytokines could also induce TOX expression in a TCR-independent manner. To generate a well-defined population of CD8 ${ }^{+} \mathrm{Tmem}$ cells, we transferred congenically marked OT-I CD $8^{+} \mathrm{T}$ cells, which express a TCR specific for the SIINFEKL peptide of OVA, into WT C57BL/6J animals followed by infection with OVA-expressing vesicular stomatitis virus (VSV-OVA; Figure 1A). We waited 60 days or longer before using these mice for subsequent experiments (referred to as VSV-OVA OT-I memory mice; Figure 1A). We isolated T cells from the spleens and lymph nodes (LNs) from VSV-OVA OT-I memory mice using negative-selection magnet-activated cell sorting (MACS) prior to ex vivo stimulation experiments (Figure 1A). This was done to ensure that cytokines act directly on T cells (30). As a negative control, we cultured bulk T cells in media alone (mock), and as a positive control, we stimulated T cells with anti-CD3/CD28 microbeads (Figure 1A). We used a combination of rIL12, rIL-15, and rIL-18 (IL-12/15/18) to induce IFN- $\gamma$ and GzmB expression in a TCR-independent manner (Figure 1A). We found that IL-12/15/18 stimulation induced PD-1 expression in OT-I Tmem cells, but the increase in expression was markedly higher after TCR ligation (Figure 1B). PD-1 frequency and median fluorescence intensity (MedFI) in OT-I Tmem increased throughout the duration of IL-12/15/18 stimulation (Figure 1B). Similarly, TCR and IL-12/15/18 stimulation induced TOX upregulation in OT-I Tmem cells (Figure 1C). Next, we measured TCF1 expression, a transcription factor needed for Tmem self-renewal that is lost in 
A
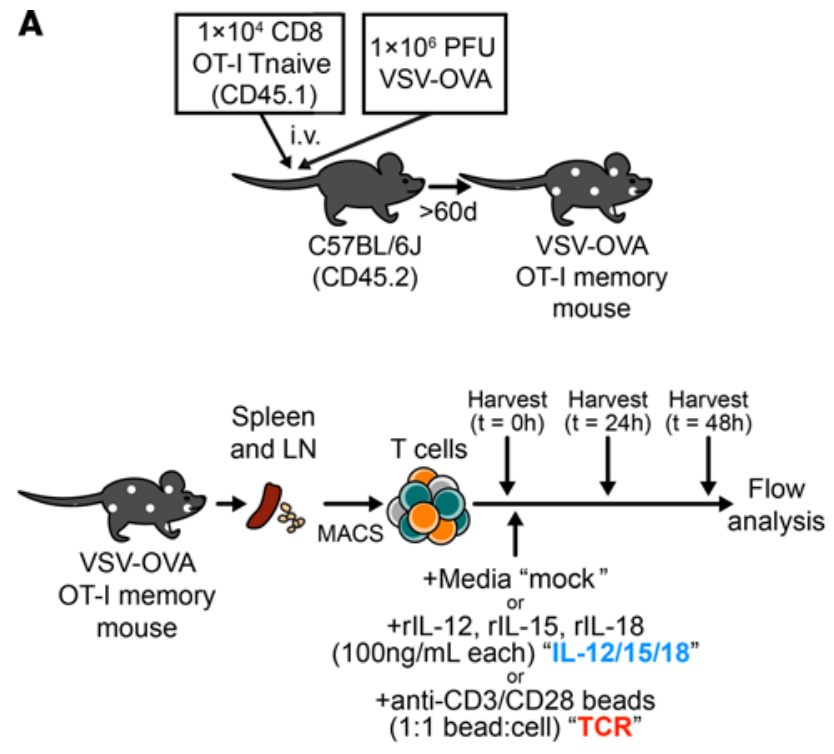

C OT-ITmem
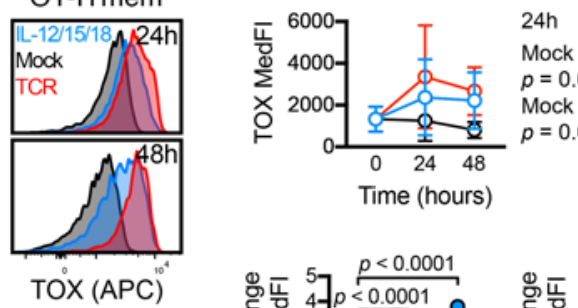

24h

Mock v ILs $\quad$ Mock v ILs Mock v TCR Mock v TCR $p=0.0030 \quad p<0.0001$

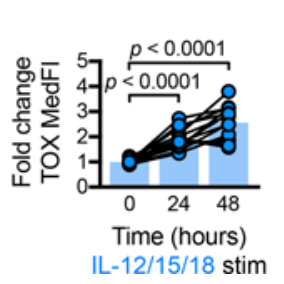

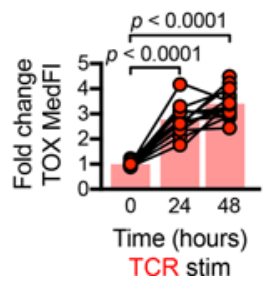

B
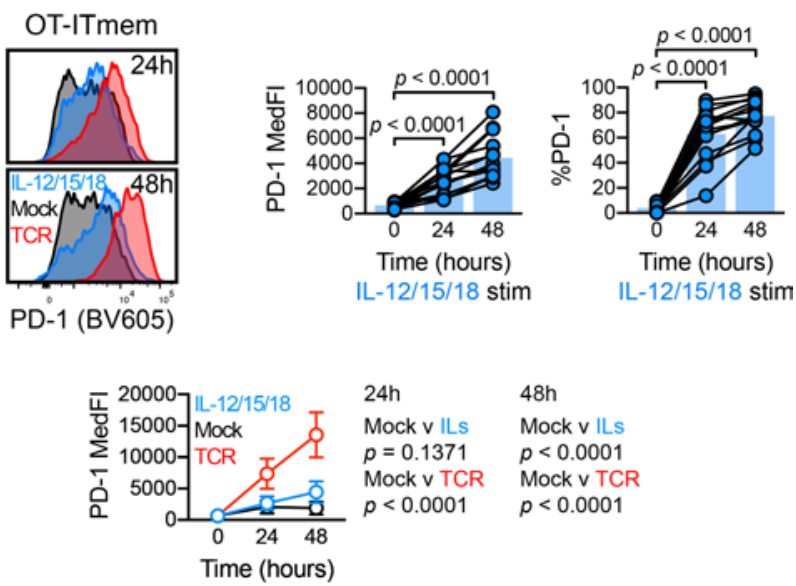

24h

Mock v ILS

$p=0.1371$

Mock v TCR $p<0.0001$

Mock v ILS

$p<0.0001$

Mock v TCR

$p<0.0001$

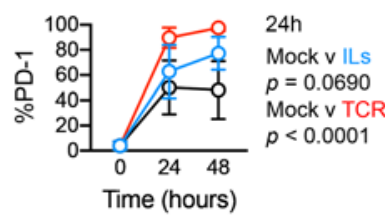

48h

Mock v ILs

$p=0.0001$

Mock v TCR

$p<0.0001$

D
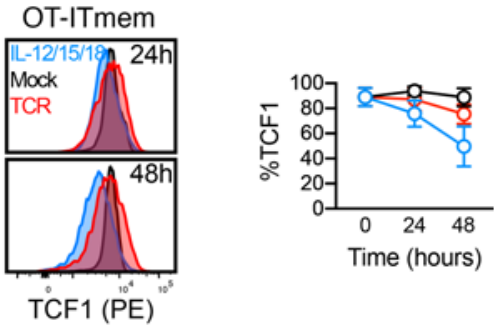

$24 \mathrm{~h}$

Mock v ILS

$p=0.0002$

Mock v TCR Mock v TCR $p=0.1297 \quad p=0.0012$

Figure 1. Cytokine stimulation induces TOX expression in murine CD8+ Tmem cells. (A) Schematic of OT-I memory mouse generation (top) and subsequent stimulation assays (bottom). OT-I Tnaive cells were transferred and expanded with VSV-OVA, then aged to stable memory contraction; after, T cells were enriched from VSV-OVA expanded OT-I memory animals and stimulated with media alone (mock), IL-12, IL-15, and IL-18 in combination (IL-12/15/18; each at $100 \mathrm{ng} / \mathrm{mL}$ ), or anti-CD3/CD28 microbeads (TCR) at an approximately 1:1 bead/cell ratio. (B and C) expression of (B) PD-1, (C) TOX, and (D) TCF1 within stimulated OT-I Tmem cells throughout experiment time course. TOX MedFI fold change in C was calculated against average TOX MedFI from mock stimulations in a subset-specific, batch-specific, and time point-specific manner. In B and C, bar chart symbols represent 1 animal at a unique time point/condition and are connected by animal identity, with bar indicating mean; the indicated statistical significances in B and C were calculated using paired $t$ tests. In B-D, symbols in line plots comparing stimulation conditions represent the mean across all animals for a specific time point/condition \pm SD; the indicated statistical significances were calculated using Mann-Whitney $U$ tests. Results from $n=14$ mice across 7 experiments are shown in B and C. Results from $n=9$ mice across 2 experiments are shown in D. TOX, thymocyte selection-associated high-mobility group box; Tmem, memory T cells; Tnaive, naive T cells; VSV-OVA, OVA-expressing vesicular stomatitis virus; PD-1, programmed cell death protein 1; MedFI, median fluorescence intensity.

terminally exhausted Tmem cells (31-34). Alongside increasing PD-1 and TOX levels, both TCR-mediated and IL-12/15/18-mediated stimulation led to significant loss of TCF1 expression in OT-I Tmem cells (Figure 1D). In sum, these data indicate that phenotypes often associated with exhaustion can be induced by TCRindependent, cytokine-mediated Tmem activation. Finally, we sought to determine whether stimulation similarly affected endogenous $\mathrm{CD}^{+}{ }^{+}$Tmem and $\mathrm{CD} 8^{+}$naive $\mathrm{T}$ (Tnaive) cells. IL-12/15/18 stimulation significantly increased TOX expression in endogenous $\mathrm{CD} 8^{+}$Tmem cells but was not observed to the same degree in $\mathrm{CD}^{+}$Tnaive cells (Supplemental Figure 1, A and B; supplemental material available online with this article; https://doi.org/10.1172/jci.insight.150744DS1). This CD8 ${ }^{+}$Tmem cell-specific response is, too, reflected in IL-12/15/18-mediated upregulation of PD-1 (Supplemental Figure 1, C and D). This is likely, in some degree, due to the different propensities of $\mathrm{T}$ cell subsets (both major and memory) to become efficiently activated by cytokines (35) and differences in cytokine receptor expression (particularly Tnaive cells, which require TCR-mediated activation to induce IL-12R and strongly increase IL-18R expression; refs. 36-38). Much akin 

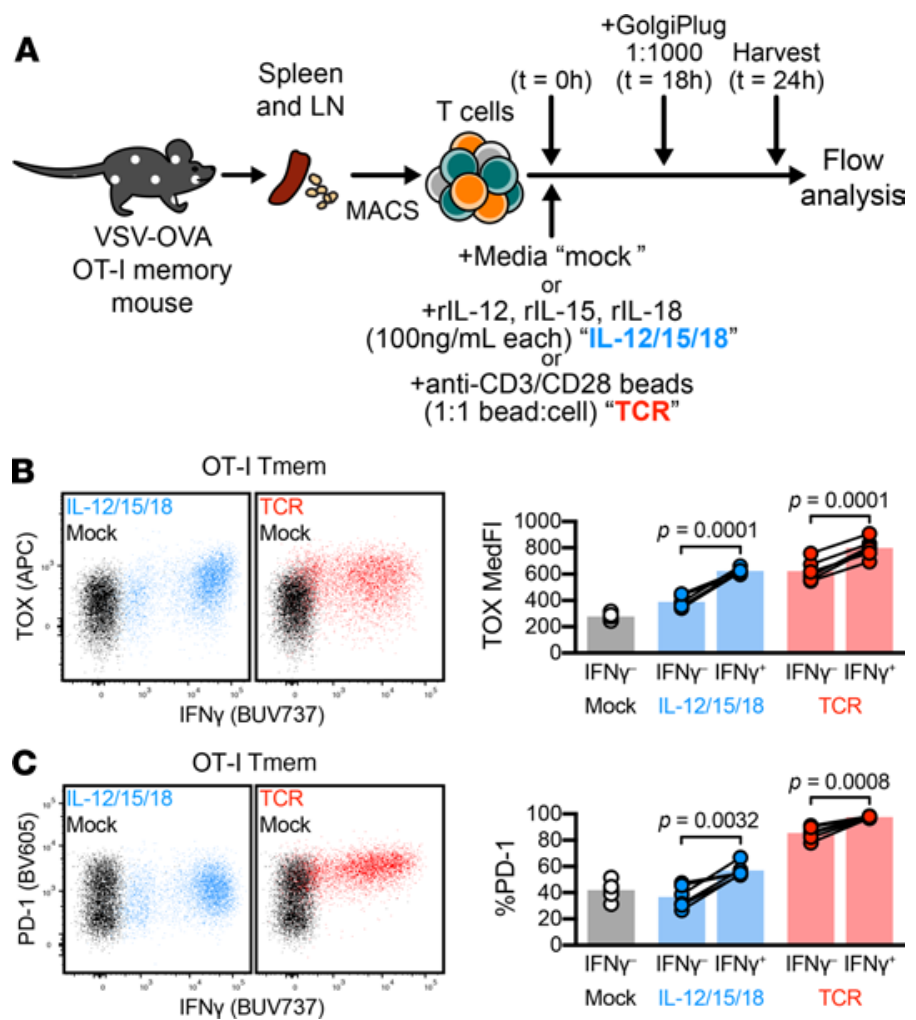

Figure 2. TOX and PD-1 expression occur in functional CD8 ${ }^{+}$T cells. ICS in tandem with TOX interrogation. (A) Experiment schematic, in which bulk T cells from VSV-OVA OT-I memory mice were stimulated (mock, black; IL-12/15/18, blue; TCR, red). Cells were treated with GolgiPlug 18 hours into stimulation and harvested for flow staining and analysis at 24 hours. (B and C) Expression of (B) TOX and (C) PD-1 in IFN- $\gamma^{+}$and IFN- $\gamma^{-}$OT-I Tmem cells. Representative plots depict cells from the same animal across different stimulation conditions. Symbols in $\mathbf{B}$ and $\mathbf{C}$ represent a $\mathrm{T}$ cell population within a unique animal with symbols connected by animal identity ( $n=6$ across 2 experiments). Bars represent mean and indicated statistical significances were calculated by paired $t$ tests. TOX, thymocyte selection-associated high-mobility group box; ICS, intracellular cytokine staining; Tmem, memory T cells; VSV-OVA, OVA-expressing vesicular stomatitis virus; PD-1, programmed cell death protein 1; TCR, T cell receptor.

to OT-I Tmem cells, TCR stimulation dramatically increased both TOX MedFI and PD-1 expression across endogenous subsets (Supplemental Figure 1, A-D), though the fold change in TOX staining intensity was most pronounced in $\mathrm{CD} 8^{+}$Tmem cells (Supplemental Figure 1A). Though IL-12/15/18 stimulation increased TOX MedFI in transgenic and endogenous $\mathrm{CD} 8^{+}$Tmem cells, it was initially to a lower degree than that of TCR-stimulated cells (Figure 1C and Supplemental Figure 1A). Because short-term TCR and IL-12/15/18 stimulation could dramatically augment TOX and PD-1 expression in CD8 ${ }^{+}$Tmem cells from VSV-OVA OT-I memory mice, we next sought to test if the upregulation of TOX and PD-1 compromises functionality.

Functional $C D 8^{+}$Tmem cells expressed TOX, PD-1, and effector proteins. We isolated T cells from VSV-OVA OT-I memory mice, as outlined in Figure 1. We stimulated T cells in the presence of Golgi inhibitors (Figure 2A) and found that OT-I Tmem cells produced substantial amounts of IFN- $\gamma$ after IL-12/15/18 or TCR stimulation; yet IFN- $\gamma$-expressing OT-I Tmem cells demonstrated higher TOX and PD-1 expression than those that failed to make IFN- $\gamma$ (Figure 2, B and C). Similarly, OT-I Tmem cells that produced GzmB after stimulation also demonstrated increased TOX and PD-1 expression (Supplemental Figure 2, A and B). Together, these data indicate that TOX and PD-1 expression levels were elevated in activated, functional CD8 ${ }^{+} \mathrm{Tmem}$ cells and suggest that TOX expression was also part of a cytokine-driven $\mathrm{T}$ cell activation program.

Induction of TOX and PD-1 was heterogeneous in $C D 8^{+}$Tmem cells. To ensure that our data were not solely reliant on OT-I T cells, we also generated gBT-I memory mice using gBT-I TCR-transgenic cells (specific for an epitope of the herpes simplex virus 2 [HSV2] glycoprotein B [gB] protein) and a recombinant, gB epitopeexpressing Listeria monocytogenes strain (L. monocytogenes $-\mathrm{gB}$; Supplemental Figure 3B). After stable contraction of TCR-transgenic Tmem cells ( $\geq 60 \mathrm{~d}$ ), we conducted stimulation assays as previously outlined (Figure 1A). IL-12/15/18-mediated or TCR-mediated stimulation led to comparable TOX upregulation in OT-I and gBT-I 
A

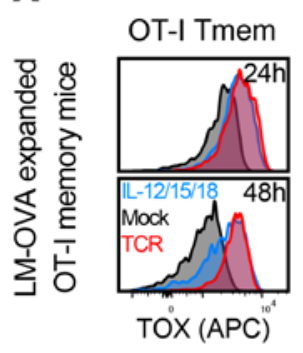

C

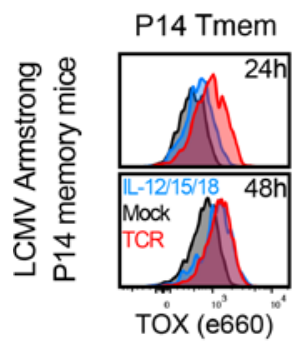

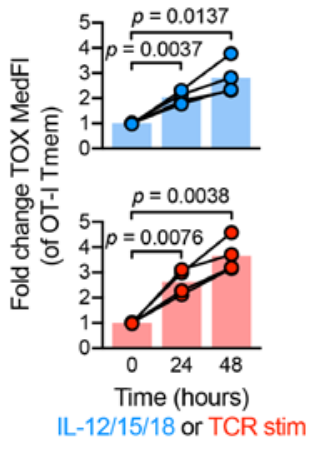

IL-12/15/18 or TCR stim
B

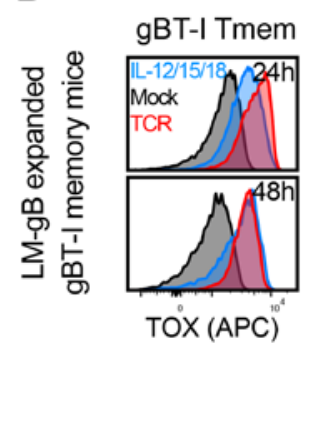

D

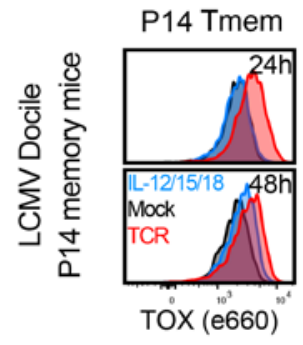

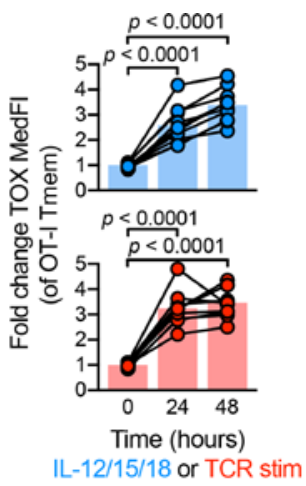

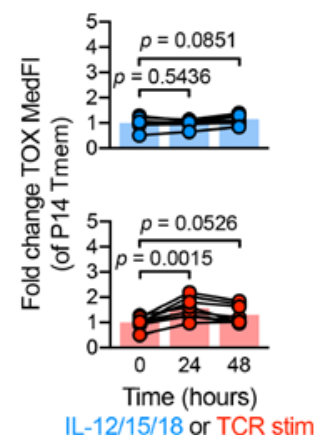

Figure 3. Cytokine-mediated TOX induction is limited in exhausted T cells. (A and B) Changes in TOX expression within L. monocytogenes-expanded TCR-transgenic Tmem cells: OT-I, specific for OVA Ag and gBT-I, specific for gB Ag. MACS-enriched T cells from L. monocytogenes-expanded OT-I or gBT-I memory mice were stimulated with media alone (mock), recombinant IL-12, IL-15, and IL-18 in combination (IL-12/15/18; each at 100 ng/mL), or anti-CD3/CD28 microbeads (TCR) at a approximately 1:1 cell/bead ratio. (A and B) Representative TOX expression and TOX MedFI fold change during stimulation in $L$. monocytogenes-primed (A) OT-I and (B) gBT-I Tmem cells. (C and D) Changes in TOX expression within LCMV-specific TCR-transgenic P14 T cells expanded by acute (Armstrong) or chronic (Docile) LCMV infection. (C and D) Representative TOX expression and TOX MedFI fold change during stimulation in P14 T cells primed by (C) LCMV Armstrong and (D) LCMV Docile. TOX MedFI fold change was calculated against average TOX MedFI within mock stimulation in a batch-specific, time point-specific manner. We calculated indicated statistical significances using paired $t$ tests. Each symbol represents a sample at a unique time point/ condition, with bars delineating mean, which are connected by donor $(n=4 \mathrm{~L}$. monocytogenes-OVA expanded OT-I memory mice across 2 experiments; $n=10 \mathrm{~L}$. monocytogenes-gB expanded gBT-I memory mice across 2 experiments; $n=17$ LCMV Armstrong-expanded P14 memory mice across 4 experiments; $n=8$ LCMV Docile-expanded P14 memory mice across 2 experiments). Mouse identities are consistent between representative flow plots within the same generation/adoptive transfer condition. TOX, thymocyte selection-associated high-mobility group box; Tmem, memory T cells; LCMV, lymphocytic choriomeningitis virus; MACS, magnet-activated cell sorting; PD-1, programmed cell death protein 1; MedFI, median fluorescence intensity; TCR, T cell receptor; gB, glycoprotein B; Ag, antigen.

Tmem cells (Figure 3, A and B). Similarly, PD-1 expression was comparable in OT-I and gBT-I Tmem cells after stimulation (Supplemental Figure 3, C and D), with a concurrent loss of TCF1 expression (Supplemental Figure 3, E and F). We next asked if altering the nature of the priming infection could affect the ability to express TOX in response to cytokine-mediated activation at the memory stage. We adoptively transferred P14 transgenic T cells, a TCR-transgenic cell line specific for LCMV gp33, followed by infection with LCMV Armstrong or Docile (Supplemental Figure 3, G and H). These LCMV strains elicit acute and chronic infections, respectively (the latter causing $\mathrm{T}$ cell dysfunction). We then stimulated $\mathrm{T}$ cells (with the same culture setup as outlined in Figure 1A) from these P14 memory mice. P14 Tmem cells from LCMV Armstrong-infected mice readily upregulated PD-1 after TCR or IL-12/15/18 stimulation (Supplemental Figure 3I). The exhausted P14 Tmem cells from LCMV Docile-infected mice already uniformly expressed PD-1 prior to stimulation, but IL-12/15/18 or TCR stimulation further increased surface PD-1 expression (via increased MedFI; Supplemental Figure 3J). P14 Tmem cells from LCMV Armstrong-infected mice increased TOX expression after TCR or IL-12/15/18 stimulation (Figure 3C). However, exhausted P14 Tmem cells from LCMV Docile-infected mice significantly increased TOX expression only after TCR stimulation (Figure 3D). Overall, P14 Tmem cells from LCMV Docile-infected mice showed a much more limited fold change in TOX MedFI compared with P14 Tmem cells from LCMV Armstrong-infected mice (Figure 3C vs. Figure 3D). Although differences between $\mathrm{CD} 8^{+}$Tmem cells from mice with acute and chronic infections are expected, the differences between gBT-I and OT-I ( $\sim$ - to 4 -fold increase in TOX expression) compared with P14 (up to $\sim 2$-fold) need to be interpreted 
with caution because the gBT-I/OT-I and P14 experiments used different TOX antibody clones (REA473 and TXRX10, respectively). Overall, our data indicate that Tmem cells that were generated by different acute infections increased TOX expression in response to proinflammatory cytokines, suggesting that this was a broadly applicable mechanism of TOX induction in the Tmem cell compartment. We next sought to determine if PD-1 and TOX upregulation in response to stimulation was similarly recapitulated in human $\mathrm{CD}^{+} \mathrm{T}$ cells.

Cytokine stimulation induced TOX and PD-1 in human Tmem cells. Using cryopreserved PBMCs from healthy, HIV-seronegative donors, we interrogated TOX and PD-1 expression by flow cytometry. We specifically gated $\mathrm{CD}^{+} \mathrm{T}$ cells by a memory and naive binary, delineating $\mathrm{CD} 8^{+}$Tnaive cells as $\mathrm{CD} 45 \mathrm{RO}^{-} \mathrm{CCR} 7^{+}$, with remaining cells as CD8 ${ }^{+}$Tmem cells (ref. 39; Figure 4A), and interrogated basal TOX and PD-1 expression between these 2 subsets (Figure 4A). Because PD-1 expression is heterogeneous in humans $(40,41)$, we measured TOX MedFI across PD-1 low-, medium-, and high-expressing events. We found that CD8 ${ }^{+}$Tmem cells with the highest PD-1 expression also demonstrated significantly elevated TOX MedFI (Figure 4B), mirroring correlations of TOX and PD-1 expression in our mouse model as well as human HCV infections (6). We next tested whether IL-12/15/18 stimulation increases PD-1 and TOX expression in T cell subsets and included mock and TCR stimulation conditions as negative and positive controls, respectively, but we also included stimulations using rIL-6, rIL-15, or rIL-12 and rIL-18 (Figure 4C). We chose these additional conditions because IL-6 activates CD8 ${ }^{+}$Tnaive cells (as evidenced by CD69 upregulation) and to discern individual activating contributions of each cytokine (Supplemental Figure 4A). Across these conditions, IL-12/15/18-mediated and TCR-mediated stimulations led to the most prominent increase of TOX staining intensity and PD- $1^{\text {hi }}$ frequency in CD8 ${ }^{+}$Tmem cells (Figure 4, D and E). We measured TCF1 expression after mock, IL-12/15/18, and TCR stimulation. A decrease in TCF1 expression accompanied an increase in TOX and PD-1 expression after IL-12/15/18 or TCR stimulation (Supplemental Figure 4B), akin to our mouse stimulation data. We further tested the degree of similarity between human and mouse T cells by measuring PD-1, TCF1, and TOX expression profiles in stimulated human $\mathrm{CD} 8^{+}$Tnaive cells. Like mouse CD $8^{+}$Tnaive cells, only TCR stimulation led to appreciable changes in TOX and PD-1 within human CD8 ${ }^{+}$Tnaive cells (Figure $4 \mathrm{~F}$ and Supplemental Figure 4C). Because IL- 6 can activate $\mathrm{CD} 8^{+}$Tnaive cells, we used this condition to determine if PD-1 and TOX expression could occur in Tnaive cells in the absence of a TCR signal. Despite inducing CD69 expression, we found that IL-6-mediated stimulation failed to increase TOX or PD-1 expression in $\mathrm{CD}^{+}$Tnaive cells (Supplemental Figure 4D). Together, these data show that CD8 ${ }^{+}$Tmem cells differentially expressed TOX, PD-1, and TCF1 at homeostasis and after both IL-12/15/18 and TCR stimulation. We next wanted to better define these changes across different Tmem subsets.

Inflammation-induced PD-1 and TOX expression occurred in most but not all CD8 $8^{+}$Tmem subsets. To test if inflammation-induced PD-1 and TOX expression differs across human $\mathrm{CD}^{+}$Tmem subsets, we used CD45RO and CCR7 staining to further delineate central memory (Tcm; CD45RO $\left.{ }^{+} \mathrm{CCR} 7^{+}\right)$, Tem $\left(\mathrm{CD} 45 \mathrm{RO}^{+} \mathrm{CCR}^{-}\right)$, and Temra (CD45RO- CCR7-) subsets (refs. 39, 42; Figure 5A). When we measured TOX, PD-1, and TCF1 expression across these subsets, we noted that a substantial fraction of $\mathrm{CD} 8^{+}$Tem events were PD- ${ }^{\text {hi }}$, and both $\mathrm{CD} 8^{+}$ Tem and Temra cells expressed elevated levels and lower levels of TOX and TCF1, respectively, at homeostasis (Figure 5B). Although this observation is in line with the initial report demonstrating TOX heterogeneity in human $\mathrm{CD}^{+}$Tmem subsets (17), it remained unknown if these CD8 ${ }^{+}$Tmem subsets are equally capable of further TOX upregulation after stimulation. We observed that TOX, PD-1, and TCF1 expression kinetics in $\mathrm{CD}^{+}$Tcm and Tem cells largely resembled one another, with both IL-12/15/18 and TCR stimulation having increased the frequency of PD- $1^{\text {hi }}$ events and TOX MedFI but having decreased TCF1 MedFI (Figure 5C). It is worth noting that although TCF1 MedFI in CD8 ${ }^{+}$Tcm cells dropped profoundly after IL-12/15/18 or TCR stimulation, the loss in frequency of TCF1-expressing cells (as defined by subjective gating) was not as pronounced as what we observed in CD8 $8^{+}$Tem cells (Supplemental Figure 5A). Although both IL-12/15/18mediated and TCR-mediated stimulation were able to significantly increase the frequency of PD- $1^{\text {hi }}$ events and lower TCF1 MedFI in CD8 ${ }^{+}$Temra cells, the degree of these changes was less pronounced than in $\mathrm{CD} 8^{+} \mathrm{Tcm}$ or Tem cells (Figure 5C). Moreover, $\mathrm{CD}^{+}$Temra cells did not significantly upregulate TOX expression after TCR stimulation. This, however, was not due to an inability to be stimulated because CD8 ${ }^{+}$Temra cells readily expressed the activation marker CD69 after cytokine-mediated or TCR-mediated stimulation (Supplemental Figure 5A). Finally, it is worth noting that when stimulated with IL-15 alone, CD8 ${ }^{+}$Tcm cells, unlike CD8 Tem and Temra cells, failed to significantly express PD-1 (Supplemental Figure 5B).

We next interrogated $\mathrm{T}$ cells with defined TCR specificity, specifically IAV-specific $\mathrm{CD} 8^{+} \mathrm{T}$ cells using HLA-A*02 tetramers loaded with the GILGFVFTL peptide (Figure $6 \mathrm{~A}$ ). We examined this $\mathrm{CD}^{+}{ }^{+}$Tmem 
A
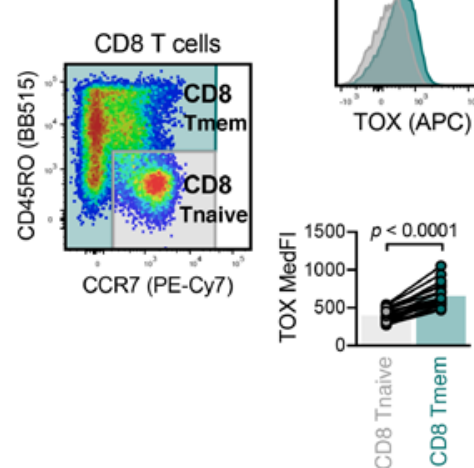

B
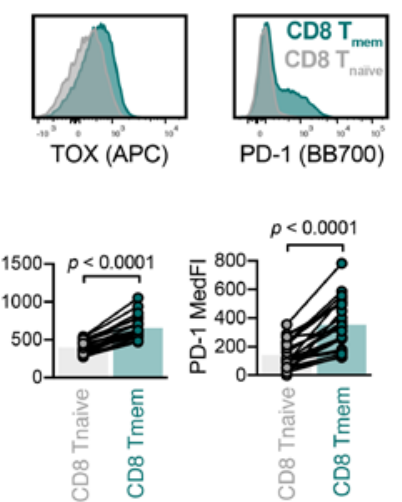

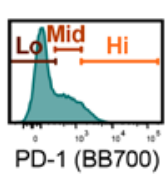

PD-1 (BB700)

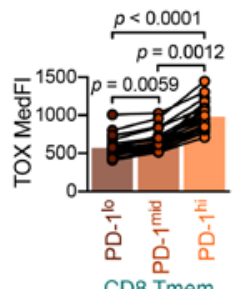

CD8 Tmem

C

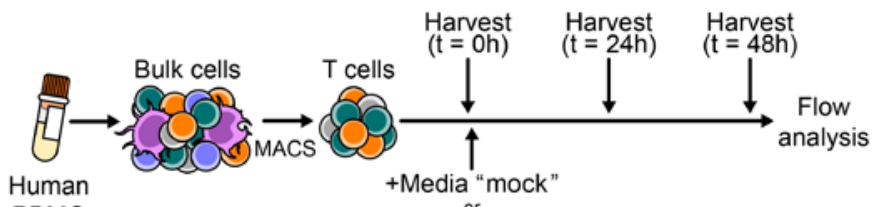

PBMC

+rIL-6 (100ng/m $/$ mL each) "IL-6"

+rlL-15 (100ng/mL each) "IL-15"

+rlL-12, rlL-18 (100ng/mL each) "IL-12/18"

+rIL-12, rlL-15, rlL-18 (100 $\mathrm{ng} / \mathrm{mL}$ each) "IL-12/15/18"

+anti-CD3/CD28 beads (1:1 bead:cell) "TCR"

D

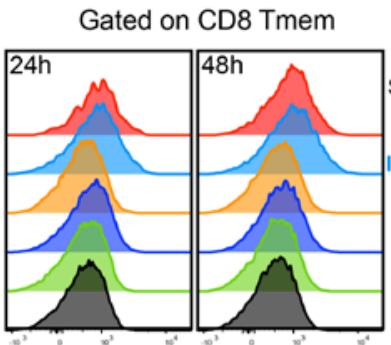

TOX (APC)

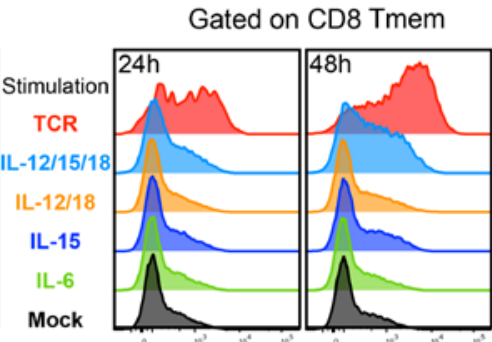

PD-1 (BB700)

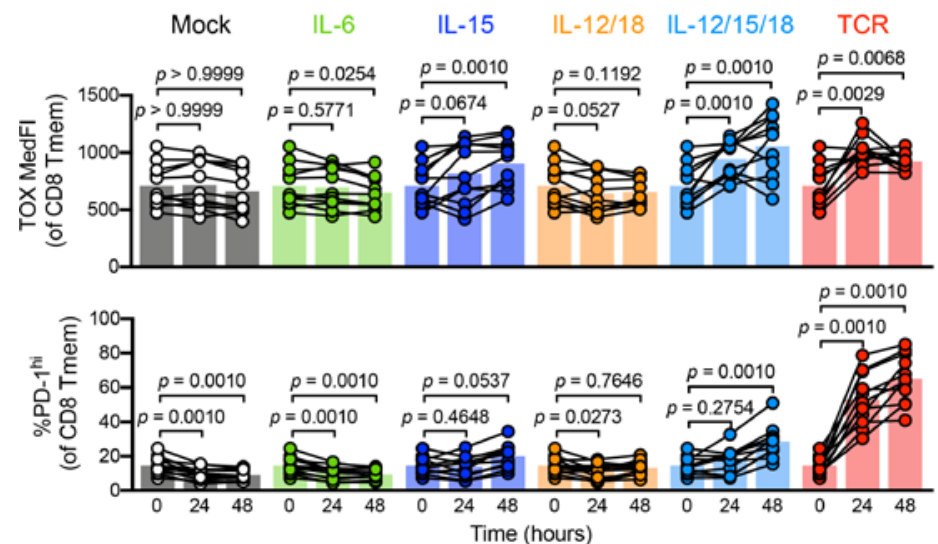

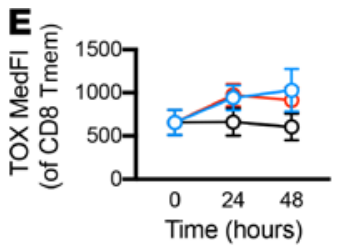

24h

Mock v ILs Mock v ILs $p<0.0001 \quad p<0.0001$ Mock v TCR Mock v TCR $p<0.0001 \quad p<0.0001$

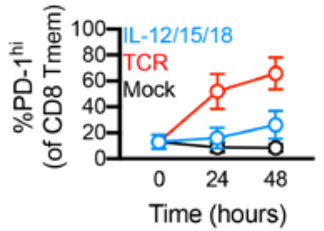

24h

Mock v ILs $p=0.0004$ Mock v TCR Mock v TCR $p<0.0001 \quad p<0.0001$
$\mathbf{F}$

Gated on CD8 Tnaive

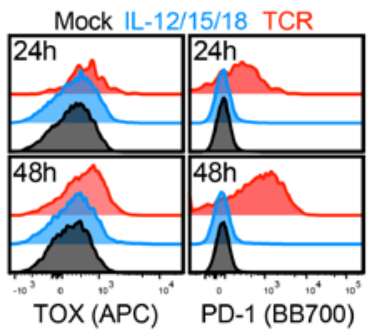

$24 \mathrm{~h}-48 \mathrm{~h}$

Mock v ILs Mock v ILs $p<0.0001 \quad p<0.0001$ Mock v TCR Mock v TCR $p<0.0001 \quad p<0.0001$

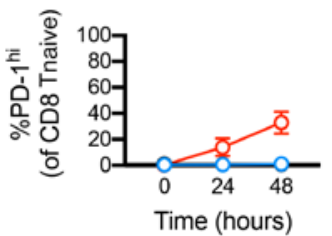

$24 \mathrm{~h}$

Mock v ILs Mock v ILs $p=0.0140 \quad p<0.0001$ Mock v TCR Mock v TCR $p<0.0001 \quad p<0.0001$

Figure 4. Inflammatory cytokines are potent inducers of TOX and PD-1 in human Tmem cells. (A) Basal expression of TOX and PD-1 in CD8 ${ }^{+}$Tmem and Tnaive cells. (B) TOX MedFI across PD-1 low-, medium-, and high-expressing CD8+ Tmem cells. (C) Schematic detailing T cell isolation from cryopreserved PBMCs and subsequent stimulation with recombinant IL-6, IL-15, IL-12/18, and IL-12/15/18 (all at $100 \mathrm{ng} / \mathrm{mL}$, each), or anti-CD3/CD28 microbeads (TCR, 1:1 bead/cell ratio) and subsequent flow interrogation. (D) TOX expression (MedFI) and PD-1hi frequency in CD8+ Tmem cells throughout stimulation time course. (E and F) Comparison of TOX MedFI and PD-1 ${ }^{\text {hi }}$ frequency in mock-, IL-12/15/18-, and TCR-stimulated (E) CD8 ${ }^{+}$Tmem and (F) CD8 ${ }^{+}$Tnaive cells. In A, B, and D-F, we calculated indicated statistical significances by (A and D) Wilcoxon's matched-pairs signed-rank tests, (B) Friedman's test with Dunn's multiple comparisons tests, or (E and $\mathbf{F}$ ) Mann-Whitney $U$ tests. In $\mathbf{A}$ and $\mathbf{D}$, each symbol represents a unique time point/treatment connected by donor with bars indicating mean (A, $n=23$ across 4 experiments; $\mathbf{D}, n=11$ across 2 experiments). In $\mathbf{E}$ and $\mathbf{F}$, each symbol represents the mean $\pm S D$ of the stimulation condition from $n=23$ donors across 4 experiments. Representative plots from $\mathbf{A}, \mathbf{D}$, and $\mathbf{F}$ are sourced from the same donor. TOX, thymocyte selection-associated high-mobility group box; Tmem, memory $T$ cells; Tnaive, naive T cells; PD-1, programmed cell death protein 1; MedFI, median fluorescence intensity; TCR, T cell receptor. 

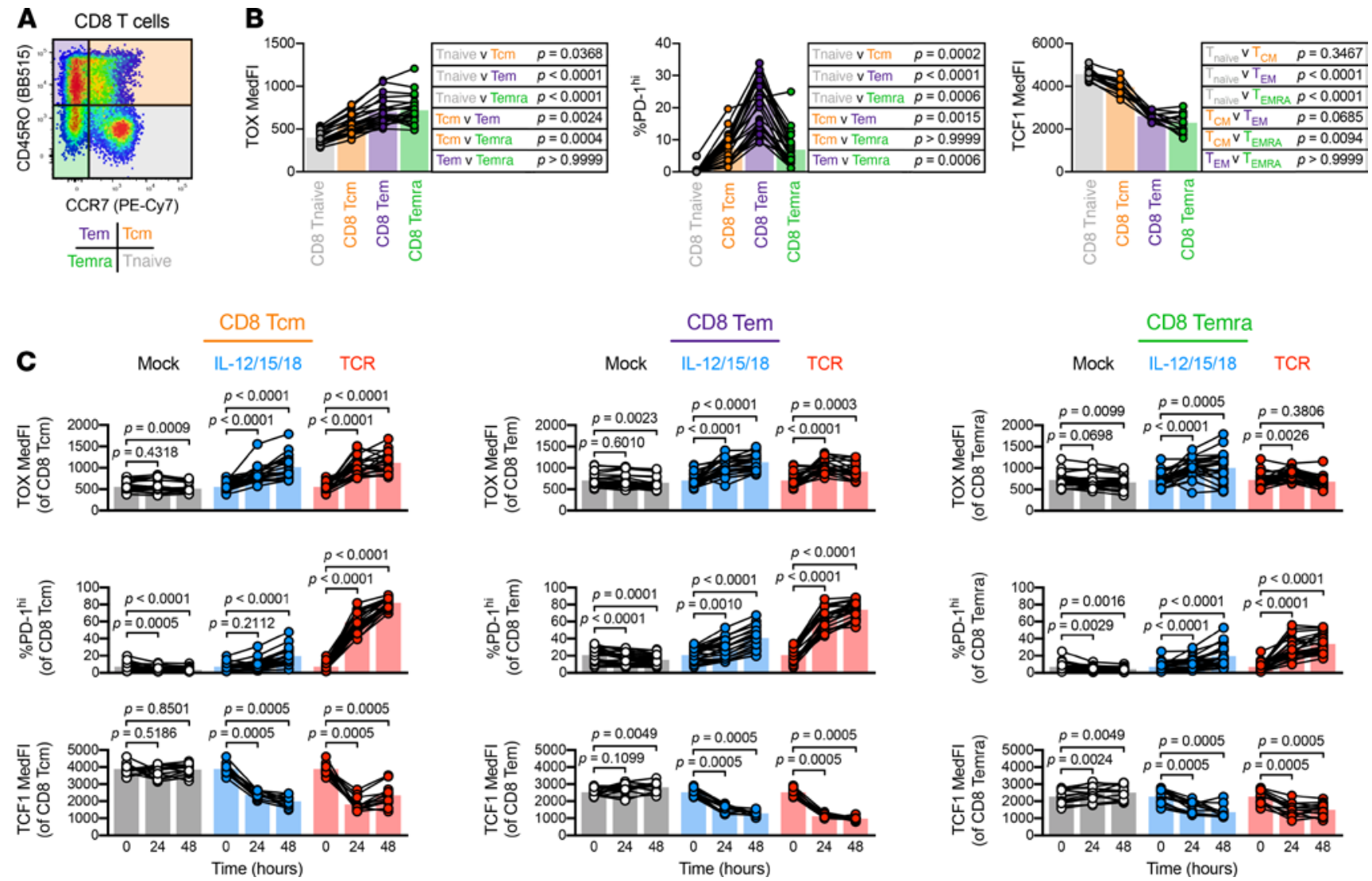

Figure 5. TOX and PD-1 upregulation are largely independent of Tmem subset. Basal and stimulation-induced TOX and PD-1 expression in CD8+ memory subsets. (A) Representative gating of CD8 ${ }^{+}$T cells into Tnaive (gray), Tcm (orange), Tem (purple), and Temra (green) subsets. (B) Basal expression levels

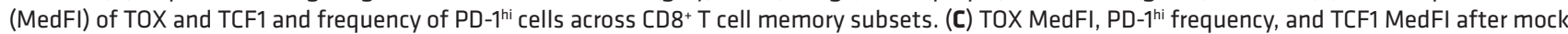
(black), IL-12/15/18 (each at $100 \mathrm{ng} / \mathrm{mL}$, blue), or TCR (1:1 bead/cell ratio, red) stimulation in CD8 ${ }^{+}$Tcm (left column), CD8 ${ }^{+}$Tem (center column), and CD8 ${ }^{+}$ Temra (right column) cells. Symbols in B and $\mathbf{C}$ represent unique samples (by time point/condition/subset) and are connected by donor identity, with bars representing mean. We determined statistical significances in B and C, respectively, using Friedman's tests and Wilcoxon's matched-pairs signed-rank tests. B and C depict $n=23$ donors across 4 experiments, except for TCF1 plots, which depict $n=12$ donors across 2 experiments. TOX, thymocyte selection-associated high-mobility group box; Tmem, memory T cells; Tnaive, naive T cells; PD-1, programmed cell death protein 1; MedFI, median fluorescence intensity; Tem, effector memory T cells; TCR, T cell receptor; Temra, CD45RA-expressing effector memory T cells; Tcm, central memory T cells.

population because these cells were reported to not express appreciable levels of TOX at homeostasis, likely owing to their Tcm phenotype (17). Within our sample set, IAV-specific $\mathrm{CD} 8^{+} \mathrm{T}$ cells were predominantly $\mathrm{Tcm}$ in half of the HLA-A ${ }^{*} 2$ PBMC donors (Figure $6 \mathrm{~A}$ ). Nevertheless, all IAV-specific $\mathrm{CD} 8^{+} \mathrm{T}$ cells were able to substantially upregulate TOX and PD-1 expression after IL-12/15/18 stimulation (Figure 6B), indicating that $\mathrm{CD} 8{ }^{+}$Tmem cells with low levels of TOX and PD-1 at homeostasis can also contribute to TOX and PD-1 heterogeneity after recent activation. Alongside testing IAV-specific $\mathrm{CD} 8^{+} \mathrm{T}$ cells, we also interrogated the effects of stimulation in mucosal associated invariant T (MAIT) cells. We selected this population because (a) MAIT cells are nonconventional $\mathrm{T}$ cells, recognizing bacterial metabolites as Ags presented on MHC-related 1 (MR1; ref. 43); (b) inflammation is necessary for sustained MAIT cell effector function (44, 45); and (c) MAIT cells are near-uniformly Tem cells when defined by CD45RO and CCR7 (46). We identified MAIT cells using MR1 tetramers loaded with the 5-OP-RU metabolite (47), which largely fell into our Tem gate (Figure 6C). Like IAV-specific CD8 ${ }^{+}$T cells, IL-12/15/18 stimulation led to substantial TOX and PD-1 upregulation in MAIT cells (Figure 6D). Because inflammation is necessary for sustained MAIT cell effector function, we asked if MAIT cells are differentially capable of responding to other cytokine combinations. Alongside IL-12/15/18, IL-15 alone or IL-12 and IL-18 in unison could significantly increase both the frequency of PD-1 ${ }^{\text {hi }}$ and TOX MedFI of MAIT cells, but not IAV-specific T cells (Supplemental Figure 6, A and B). Together, these data indicate that cytokine-driven activation programs were conserved across conventional and innate-like $\mathrm{T}$ cells.

Cytokine stimulation-induced PD-1 expression was independent of TOX. Finally, because PD-1 and TOX upregulation appeared tightly associated after cytokine-driven activation, we next asked if this association is mechanistic 
A

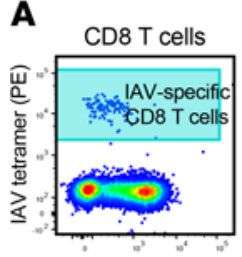

CCR7 (PE-Cy7)

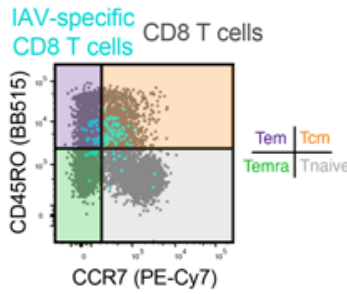

CCR7 (PE-Cy7)

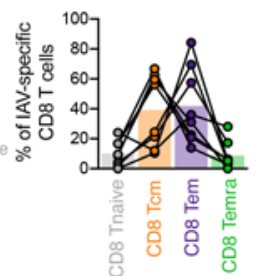

IL-12/15/18

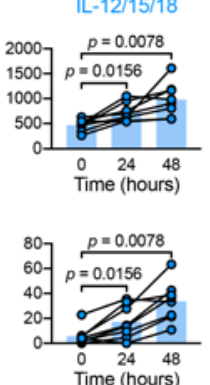

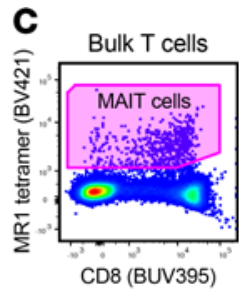

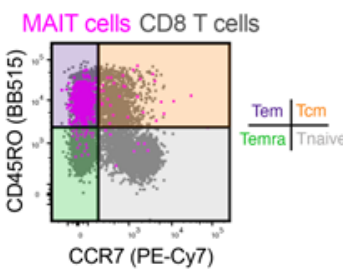

Mock

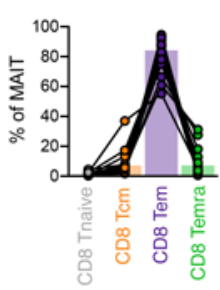

IL-12/15/18
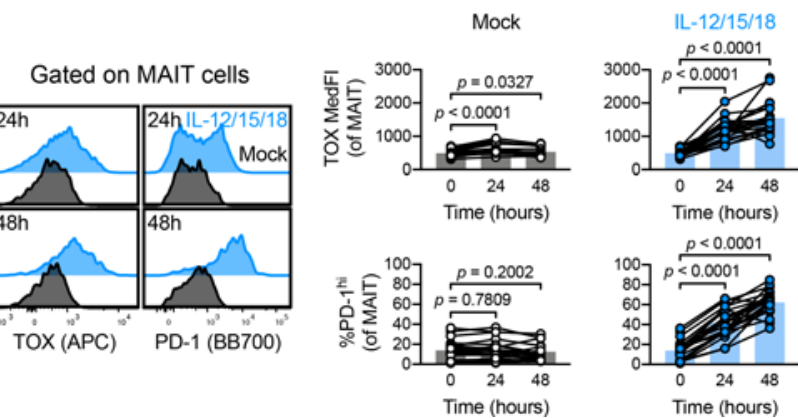

Figure 6. Stimulation induces TOX and PD-1 expression in conventional and innate-like T cells. TOX and PD-1 induction in IAV-specific CD8+ T cells. (A) Gating and memory phenotyping of IAV-specific CD8+ T cells. (B) Induction of TOX and PD-1 in IAV- specific CD8 ${ }^{+}$T cells by mock (black) or IL-12/15/18 (each at 100 ng/ mL, blue) stimulation. (C and D) TOX and PD-1 induction in MAIT cells. (C) Gating and memory phenotyping of MAIT cells. (D) Induction of TOX and PD-1 in MAIT cells by mock (black) or IL-12/15/18 (each at $100 \mathrm{ng} / \mathrm{mL}$, blue) stimulation. Representative plots are sourced from the same donor. Symbols represent unique samples (by time point/condition/subset) and are connected by donor identity, with bars representing mean. We determined statistical significances in B and D using Wilcoxon's matched-pairs signed-rank tests. A and B depict $n=8$ donors across 2 experiments; C and $\mathbf{D}$ depict $n=23$ donors across 4 experiments. TOX, thymocyte selection-associated high-mobility group box; PD-1, programmed cell death protein 1; IAV, influenza A virus; MAIT, mucosal associated invariant T.

in nature. If TOX is necessary for PD-1 expression, then it would allow the use of surface-expressed PD-1 as a surrogate for intracellularly expressed TOX. TOX expression appears to drive PD-1 expression in a number of contexts because exhausted Tmem cells dramatically downregulate PD-1 after TOX deletion or knockdown (6, 13, 15, 48). Conversely, T cell transduction with TOX-encoding constructs leads to PD-1 upregulation (13-15, 48). Although TOX controls PD-1 expression during exhaustion, the role of TOX is less clear in activation. To dissect the function of TOX in stimulation-mediated PD-1 upregulation, we used WT and Tox ${ }^{-1}$ P14 Tmem cells. To generate these P14 Tmem cells, we adoptively transferred WT or KO P14 T cells into C57BL/6J hosts, which we subsequently infected with LCMV Armstrong to form a Tmem population (Supplemental Figure 7A). To determine if TOX deficiency alters stimulation-induced PD-1 upregulation, we cultured MACS-isolated $\mathrm{T}$ cells from WT and Tox $x^{--}$P14 memory mice (28 days after LCMV Armstrong infection) in the presence of mock, IL-12/15/18, or TCR stimulation (Supplemental Figure 7A). Both WT and Tox ${ }^{-1-}$ P14 Tmem cells increased PD-1 expression after IL-12/15/18 or TCR stimulation (Figure 7, A-C). Together these data indicate that TOX alone was not necessary for PD-1 upregulation in cytokine-stimulated CD8 ${ }^{+}$Tmem cells and suggest other transcription factors were sufficient to drive PD-1 expression in the absence of TOX.

\section{Discussion}

TOX has been foremost studied in TCR-mediated exhaustion of mouse CD8 ${ }^{+} \mathrm{T}$ cells in the context of tumor or chronic infection $(6,13,14)$. A recent study reported TOX expression in functional circulating human CD8 Tmem cells, suggesting TOX expression does not necessarily dictate dysfunction $(17,18)$, which led to the speculation that TOX may have distinct roles across species, specifically mice and humans (19). Alternatively, TOX expression heterogeneity in humans may simply reflect the more complex environment that human $\mathrm{T}$ cells are exposed to in everyday life, which may not be readily appreciable in specific pathogen-free mice, such as routine inflammatory events in barrier tissues. Thus, we asked if proinflammatory cues could be sufficient to increase TOX expression and contribute to TOX heterogeneity. Although inflammation has been previously shown to enhance TCR-mediated TOX upregulation (in a VEGF-A-dependent manner that necessitates initial TCR signaling; ref. 49), our findings are, to the best of our knowledge, the first to demonstrate TOX expression in the absence of agonist TCR signals. Transient IL-12/15/18 and TCR stimulation increased PD-1 and TOX expression in most CD8 ${ }^{+}$Tmem cells. In mouse, dysfunctional P14 Tmem cells from LCMV Docile-infected mice still increased surface PD-1 expression after TCR stimulation, whereas IL-12/15/18 had little to no effect 
A
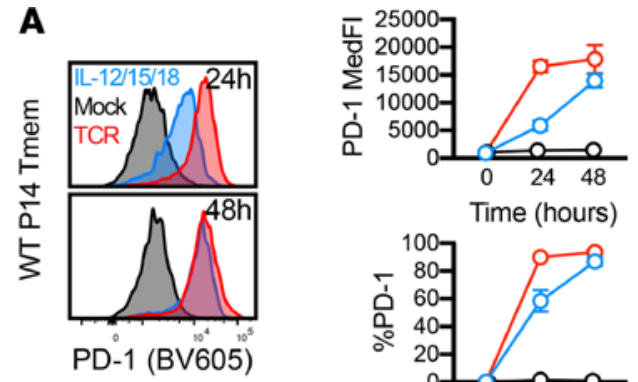

Time (hours)

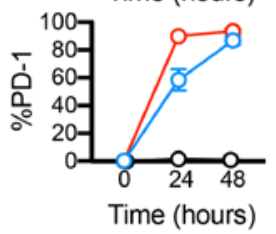

B

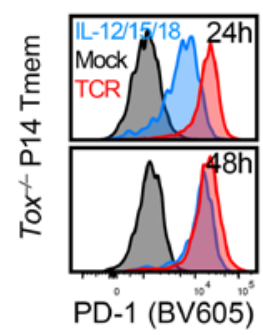

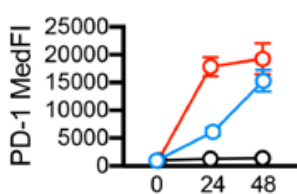

Time (hours)

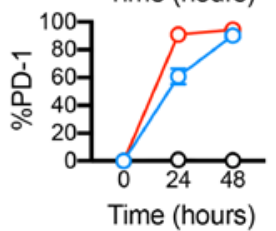

24h

Mock v ILs

$p<0.0001$

Mock v TCR

$p<0.0001$

24h

Mock v ILs

$p<0.0001$

Mock v TCR

$p<0.0001$

24h

Mock v ILs

$p<0.0001$

Mock v TCR

$p<0.0001$

24h

Mock v ILs

$p<0.0001$

Mock v TCR

$p<0.0001$ 48h

Mock v ILs

$p<0.0001$

Mock v TCR

$p<0.0001$

$48 \mathrm{~h}$

Mock v ILs

$p<0.0001$

Mock v TCR

$p<0.0001$

48h

Mock v ILS

$p<0.0001$

Mock v TCR

$p<0.0001$

48h

Mock v ILs

$p<0.0001$

Mock v TCR

$p<0.0001$
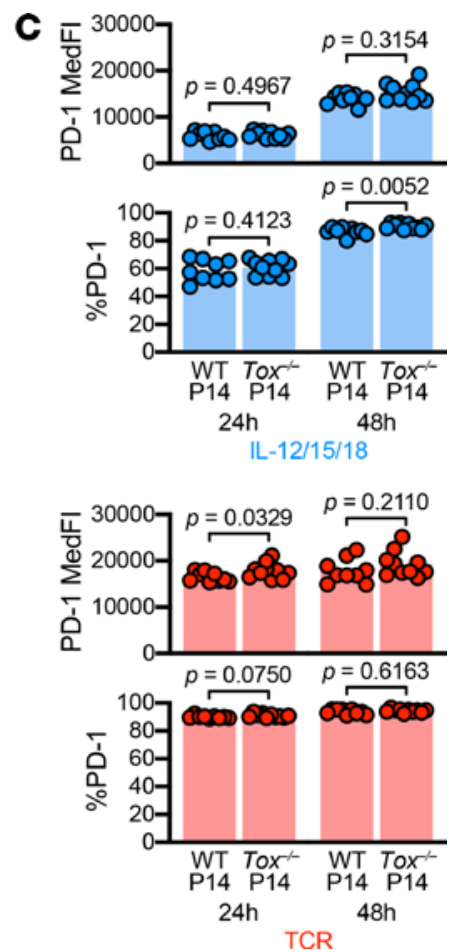

Figure 7. TOX deficiency does not abrogate stimulation-induced PD-1 expression. (Stimulation-induced PD-1 expression in WT and Tox $x^{-/-}$P14 Tmem cells. T cells were stimulated with media alone (mock), recombinant IL-12, IL-15, and IL-18 in combination (IL-12/15/18 or ILs; each at $100 \mathrm{ng} / \mathrm{mL}$ ), or with anti-CD3/ CD28 microbeads at an approximately 1:1 cell/bead ratio (TCR). (A and B) PD-1 MedFI and expression frequencies in A WT or B Tox ${ }^{-1-}$ P14 Tmem over stimulation time course. (C) Comparison of PD-1 MedFI and expression frequencies between IL-12/15/18 (left) or TCR (right) stimulated WT and Tox ${ }^{-1-}$ P14 Tmem cells. All indicated statistical significances were calculated using Mann-Whitney $U$ tests. Symbols in $\mathbf{A}$ and $\mathbf{B}$ represent the mean $\pm S D$ from all animals at a specific time/condition; and symbols in C represent stimulated P14 Tmem populations within a single animal ( $n=9$ WT P14 recipients and $n=10$ Tox ${ }^{-1-}$ P14 recipient across 2 experiments). TOX, thymocyte selection-associated high-mobility group box; Tmem, memory T cells; PD-1, programmed cell death protein 1; MedFI, median fluorescence intensity; TCR, T cell receptor.

on TOX expression. Similarly, human Temra cells showed limited to no increase in TOX expression after exposure to IL-12/15/18. The underlying mechanisms will require further investigation, but one could speculate that the cytokine stimulation was simply not potent enough to further enhance the already ongoing effector or activation program in these 2 Tmem subsets. The notion that not only TOX but also PD-1 expression can indicate an ongoing effector or activation program in $\mathrm{CD}^{+} \mathrm{T}$ cells is important because PD-1 and (now also) TOX are used as biomarkers of T cell exhaustion (50-52). Of note, certain features of general activation programs of $\mathrm{CD}^{+}$Tmem cells appear to be well conserved and have also been reported as transcriptomic overlap of tissue-resident, recently activated, and exhausted $\mathrm{CD}^{+} \mathrm{T}$ cells (53). Although infection parameters and inflammatory events are well defined in mouse model studies, most human studies remain agnostic in regard to the infection and activation history of Ag-specific T cells. This in turn makes it difficult to correctly interpret the underlying reason for expression of PD-1 and TOX by human T cells.

Our data emphasize the need for conservative interpretation of TOX in regard to activation and exhaustion and also caution against interpreting TOX expression purely through the lens of recent TCR-mediated activation. TOX expression is predictive of T cell exhaustion and unfavorable outcome in hepatocellular carcinoma animal models and clinical samples (54), in line with the paradigm of TOX-mediated, TCR-dependent $\mathrm{T}$ cell dysfunction. However, other studies have yielded contradictory data. Meta analyses of TOX expression in breast cancers reported TOX levels paradoxically correlating with increased immune cell function and favorable prognosis (55). This is perplexing, as in tumors, TOX expression is associated with T cell dysfunction (6, 13, 14). This discrepancy could be in part explained by TOX upregulation during activation, akin to what we observed during $\mathrm{T}$ cell activation in TCR-dependent and -independent stimulations. Thus, our data stress that all possible activation pathways of TOX and PD-1 induction must be considered before interpreting TOX as a biomarker of T cell dysfunction. A well-done human study (17) that interrogated TOX heterogeneity found elevated TOX in CMV-specific and EBV-specific $\mathrm{CD} 8^{+}$Tmem cells and hypothesized that recent viral reactivation provide cognate Ag to facilitate TCR-mediated upregulation of TOX. This is certainly a plausible explanation, 
but our data highlight the need to also consider recent exposure to inflammation as a critical parameter affecting TOX expression. Conventional CD8 ${ }^{+}$Tem and Temra cells (the predominant phenotype of CMV-specific and EBV-specific CD8 ${ }^{+} \mathrm{T}$ cells) express elevated levels of TOX basally; Tcm (including IAV-specific CD8 ${ }^{+} \mathrm{T}$ cells) and innate-like MAIT cells can, too, upregulate TOX expression after inflammation-mediated activation. Importantly, our data highlight that this mechanism of TOX expression was conserved across species, conventional CD8 ${ }^{+}$Tmem subsets, and innate-like MAIT cells.

Because proinflammatory cytokines can concurrently induce TOX and PD-1 expression, these signals may drive TOX heterogeneity in other contexts. P14 tissue-resident Tmem cells show increased Tox expression at homeostasis, which is observed 90 days after priming with LCMV Armstrong (20). Because the acute infection is cleared well before this time point, it is unlikely that continued TCR signaling by cognate Ag drives this phenotype, despite elevated transcripts encoding mediators of TCR signaling (20). However, IL-15 is likely present within the tissue microenvironment. IL-15 is implicated in Trm cell maintenance $(25,26)$, and transcriptional profiles indicative of IL-15/STAT5 signaling are detected in human Trm $(23,24)$. Thus, IL-15 in tissue microenvironments may also contribute to TOX heterogeneity. Future work will be necessary to dissect the role of these inflammatory cues versus other signals that can shape Trm phenotype, such as costimulation and tonic TCR signaling (21).

Previous studies have demonstrated that TOX ablation or knockdown leads to PD-1 downregulation in models of exhaustion $(6,13,15,48)$, and, conversely, introduction of TOX-expressing constructs enhances PD-1 expression $(15,48)$. Similarly, our data showed a close correlation in regards to TOX and PD-1 expression levels, but we found that PD-1 expression could be induced in stimulated Tox ${ }^{-1}$ P14 Tmem cells. Of note, these Tox ${ }^{-1}$ P14 Tmem cells lacked exon 5, which abrogates the ability to function as a transcription factor, but the truncated protein is still expressed and detected by the TOX antibody. Alfei et al. (6) previously showed that the early wave of effector cells formed from Tox ${ }^{-1-}$ Tnaive cells express significant levels of PD-1 independently of functional TOX. However, TOX is required for the expression of high levels of PD-1 at later stages, once the initial population of exhausted effector $\mathrm{T}$ cells are replaced by a proliferation competent TCF1 progenitor population (31). Together, these data suggest that the long-term expression of PD-1 requires TOX, but the activation-induced expression of PD-1 is TOX-independent. In the absence of TOX, PD-1 expression could be driven by TOX2, which can induce PD-1 expression in CD8 $8^{+} \mathrm{T}$ cells $(15,48)$; however, it remains unclear if TOX2 is also upregulated by transient TCR-mediated or cytokine-mediated stimulation. Similarly, how different activating signals integrate to regulate TOX expression also requires further studies: although inflammatory cues increase TOX expression in Tmem cells, increased IL-12 signaling during the priming of Tnaive cells has been shown to limit subsequent TOX expression at steady state $(56,57)$.

Overall, our data suggest that the mechanisms that regulate TOX expression, both at homeostasis and after transient TCR or cytokine stimulation, were remarkably similar and quite possibly highly conserved between humans and mice. Our data further highlight the need to consider TOX and PD-1 expression as prominent indicators of ongoing activation and effector programs in Tmem instead of exclusive biomarkers of exhaustion.

\section{Methods}

Mice. All animals were maintained in specific pathogen-free facilities and infected in modified pathogenfree facilities. Experimental groups were nonblinded; animals were randomly assigned to experimental groups; and no specific method was used to calculate sample sizes.

We purchased 6-week-old female C67BL/6J mice from The Jackson Laboratory; Tox $x^{-1}$ P14 mice (P14 $T_{0} x^{\text {tmlc(KOMP)Wtsi }} ; M x^{\text {Cre }} ;$ Rosa26-STOP-eYFP) were generated as previously described (6). WT and Tox ${ }^{-1-} \mathrm{P} 14$ mice, OT-I mice, and gBT-I mice were maintained on CD45.1 congenic backgrounds. We euthanized mice in accordance with institutional protocols and subsequently collected spleens and LNs for experimentation.

Development of memory mice. We prepared a single-cell suspension of LN cells that were harvested from female OT-I, P14, or gBT-I mice by mechanically passing LN tissue through a 70 to $100 \mu \mathrm{m}$ strainer. To enrich transgenic T cells, we used MACS with a CD8 Negative Selection Kit (Miltenyi Biotec).

For OT-I memory mice, we adoptively transferred $1 \times 10^{4}$ OT-I T cells in sterile $1 \times$ PBS i.v. per C57BL/6J recipient and subsequently infected recipients i.v. with 1 to $2 \times 10^{7} \mathrm{PFU}$ VSV-OVA or $4 \times 10^{3}$ CFU OVA-expressing L. monocytogenes (L. monocytogenes-OVA). For gBT-I memory mice, we adoptively transferred $5 \times 10^{4}$ gBT-I T cells i.v. and subsequently infected recipient mice i.v. with or $4 \times 10^{3} \mathrm{CFU}$ HSV2 gB-expressing L. monocytogenes (L. monocytogenes-gB). We allowed 60 days or longer to pass after initial VSV or L. monocytogenes infections before assaying tissues. 
For P14 memory mice, we adoptively transferred $2 \times 10^{3}$ WT P14 T cells i.v. and subsequently infected recipient mice i.v. with $2 \times 10^{5} \mathrm{PFU}$ LCMV Armstrong clone (LCMV Arm.) or $2 \times 10^{6} \mathrm{PFU}$ LCMV Docile clone (LCMV Doc.). For Tox ${ }^{\prime-}$ P14 memory mice, we adoptively transferred $2 \times 10^{3}$ Tox $^{-1-}$ P14 memory mice and subsequently infected with $2 \times 10^{5}$ PFU LCMV Arm.; we allowed 28 days to pass after initial LCMV infection before assaying tissues.

$T$ cell isolation and in vitro stimulation. We harvested spleen and LN from memory mice and mechanically prepared single-cell suspensions. We thawed approximately $4 \times 10^{7}$ cryopreserved PBMC in human RP10 media (RPMI1640 supplemented with 10\% FBS, 2 mM L-glutamine, $100 \mathrm{U} / \mathrm{mL}$ penicillin-streptomycin). To enrich bulk $\mathrm{T}$ cells from single-cell suspensions, we respectively used mouse-specific and human-specific $\mathrm{T}$ cell negative isolation MACS (STEMCELL Technologies). We plated 0.5 to $1 \times 10^{6} \mathrm{~T}$ cells per well in 96-well V-bottom tissue culture plates. We cultured cells in human RP10 or mouse RP10 media (RPMI 1640 supplemented with $10 \%$ FBS, $2 \mathrm{mM}$ L-glutamine, $100 \mathrm{U} / \mathrm{mL}$ penicillin-streptomycin, $1 \mathrm{mM}$ sodium pyruvate, $0.05 \mathrm{mM} \beta$-mercaptoethanol, and $1 \mathrm{mM}$ HEPES). To stimulate cells, we cultured mouse $\mathrm{T}$ cells in mouse RP10 with rIL-12, rIL-15, and rIL-18 (each at $100 \mathrm{ng} / \mathrm{mL}$; BioLegend), with Dynabeads mouse T-Activator (Thermo Fisher) anti-CD3/CD28 beads (at a 1:1 bead/cell ratio) or media alone. For human T cell stimulations, we used human RP10 media with combinations of rIL-6 (BioLegend), rIL-12, rIL-15, and/or rIL-18 (each at $100 \mathrm{ng} / \mathrm{mL}$; Peprotech), with Dynabeads human T-Activator (Thermo Fisher) anti$\mathrm{CD} 3 / \mathrm{CD} 28$ beads (at a 1:1 bead/cell ratio) or RP10 alone. We cultured cells at $37^{\circ} \mathrm{C}, 5 \% \mathrm{CO}_{2}$, sampling cells at 0,24 , and 48 hours for flow staining. For intracellular cytokine staining, we added GolgiPlug (BD Biosciences) at a 1:1000 dilution 8 hours prior to cell harvest.

Flow cytometric analysis. We conducted all flow staining for mouse and human $\mathrm{T}$ cells on ice and at room temperature, respectively. All mouse and human flow panel reagent information, stain conditions, and gating are included in Supplemental Figures 8-11 and Supplemental Tables 1-6. We conducted LIVE/DEAD fixable aqua or blue viability dye (Thermo Fisher) (AViD or BViD, respectively) or Zombie Near-IR viability dye (NIRViD) (BioLegend) staining in $1 \times$ PBS. For surface staining, we utilized FACS Wash $(1 \times$ PBS supplemented with $2 \%$ FBS and $0.2 \%$ sodium azide) as the stain diluent. For all TOX staining panels, we fixed cells with the FOXP3 Fixation/Permeabilization Buffer Kit (Thermo Fisher) and conducted intranuclear stains using the FOXP3 Permeabilization Buffer (Thermo Fisher) as diluent. To minimize day-to-day variation for TOX staining, we conducted all intracellular stains within a batch (0-, 24-, and 48-hour samples) at the same time. We resuspended cells in FACS Wash and acquired events on a FACSymphony A5 and LSRFortessa cell analyzers (BD Biosciences), which we analyzed using FlowJo v10 (BD Biosciences). We conducted statistical testing using Prism v8 (GraphPad).

Statistics. We used 2-tailed paired $t$ tests, Mann-Whitney $U$ tests, Wilcoxon's matched-pairs signed-rank tests, as well as Friedman's tests with Dunn's multiple comparisons tests.

Study approvals. Mouse protocols and experimentation conducted the Fred Hutchinson Cancer Research Center were approved by and in compliance with the ethical regulations of the Fred Hutchinson Cancer Research Center's IACUC. Experiments performed at the Technical University of Munich were in compliance with institutional and governmental regulations in Germany and approved by the veterinarian authorities of the Regierung von Oberbayern in Germany.

Twenty-three healthy, HIV-uninfected adults were recruited by the Seattle HIV Vaccine Trials Unit (Seattle, Washington, USA) as part of the study "Establishing Immunologic Assays for Determining HIV-1 Prevention and Control." These samples are also known as the Seattle Area Control Cohort. All participants were provided and signed informed consent, and the Fred Hutchinson Cancer Research Center IRB approved the study protocol.

\section{Author contributions}

NJM, JB, DZ, and MP designed research studies. NJM, JB, and AKT conducted the experiments and acquired the data. NJM, JB, AKT, DZ, and MP analyzed the data. NJM, JB, DZ, and MP wrote the manuscript, and NJM, JB, AKT, DZ, and MP edited the manuscript to its final format. NJM. conceived the initial study and was therefore designated the first-listed co-first author for this study.

\section{Acknowledgments}

We thank Andrea Schietinger for helpful discussions and critical review of the manuscript. We also thank the Prlic lab, especially Jami Erickson, Florian Mair, Marie Frutoso, and Veronica Davé, for critical review of the 
manuscript. This work was supported by NIH grant R01 AI123323 (to MP) and National Cancer Institute Grant F99 CA245735 (to NJM). NJM is a Leslie and Pete Higgins Achievement Rewards for College Scientists Fellow and Dr. Nancy Herrigel-Babienko Memorial Scholar. DZ and JB were supported by a European Research Council consolidator grant (ToCCaTa) and by the German Research Foundation (SFB1054 and SFB1371).

Address correspondence to: Dietmar Zehn, Division of Animal Physiology and Immunology, School of Life Sciences Weihenstephan, Technical University of Munich, Weihenstephaner Berg 3/I, 85354 Freising, Germany. Email: dietmar.zehn@tum.de. Or to: Martin Prlic, Fred Hutchinson Cancer Research Center, 1100 Fairview Ave. N, Seattle, Washington 98109, USA. Email: mprlic@fredhutch.org.

1. Zajac AJ, et al. Viral immune evasion due to persistence of activated T cells without effector function. $J$ Exp Med. 1998;188(12):2205-2213.

2. Pauken KE, Wherry EJ. Overcoming T cell exhaustion in infection and cancer. Trends Immunol. 2015;36(4):265-276.

3. Schietinger A, Greenberg PD. Tolerance and exhaustion: defining mechanisms of T cell dysfunction. Trends Immunol. 2014;35(2):51-60.

4. Goepfert PA, et al. A significant number of human immunodeficiency virus epitope-specific cytotoxic T lymphocytes detected by tetramer binding do not produce gamma interferon. J Virol. 2000;74(21):10249-10255.

5. Zehn D, et al. Immune-surveillance through exhausted effector T-cells. Curr Opin Virol. 2016;16:49-54.

6. Alfei F, et al. TOX reinforces the phenotype and longevity of exhausted T cells in chronic viral infection. Nature. 2019;571(7764):265-269.

7. Lee PP, et al. Characterization of circulating T cells specific for tumor-associated antigens in melanoma patients. Nat Med. 1999;5(6):677-685.

8. Utzschneider DT, et al. T cells maintain an exhausted phenotype after antigen withdrawal and population reexpansion. Nat Immunol. 2013;14(6):603-610.

9. Speiser DE, et al. T cell differentiation in chronic infection and cancer: functional adaptation or exhaustion? Nat Rev Immunol. 2014;14(11):768-774.

10. Barber DL, et al. Restoring function in exhausted CD8 T cells during chronic viral infection. Nature. 2006;439(7077):682-687.

11. Schietinger A, et al. Tumor-specific T cell dysfunction is a dynamic antigen-driven differentiation program initiated early during tumorigenesis. Immunity. 2016;45(2):389-401.

12. Blackburn SD, et al. Selective expansion of a subset of exhausted CD8 T cells by alphaPD-L1 blockade. Proc Natl Acad Sci U S A. 2008;105(39):15016-15021.

13. Scott AC, et al. TOX is a critical regulator of tumour-specific T cell differentiation. Nature. 2019;571(7764):270-274

14. Khan O, et al. TOX transcriptionally and epigenetically programs CD8+ T cell exhaustion. Nature. 2019;571(7764):211-218.

15. Seo H, et al. TOX and TOX2 transcription factors cooperate with NR4A transcription factors to impose CD $8^{+} \mathrm{T}$ cell exhaustion. Proc Natl Acad Sci U S A. 2019;116(25):12410-12415.

16. Yao C, et al. Single-cell RNA-seq reveals TOX as a key regulator of CD8 ${ }^{+} \mathrm{T}$ cell persistence in chronic infection. Nat Immunol. 2019;20(7):890-901.

17. Sekine T, et al. TOX is expressed by exhausted and polyfunctional human effector memory CD8 ${ }^{+}$T cells. Sci Immunol. 2020;5(49):eaba7918.

18. Galletti G, et al. Two subsets of stem-like $\mathrm{CD}^{+}$memory T cell progenitors with distinct fate commitments in humans. Nat Immunol. 2020;21(12):1552-1562.

19. Utzschneider DT, Kallies A. Human effector T cells express TOX-Not so “TOX”ic after all. Sci Immunol. 2020;5(49):eabc8272.

20. Kurd NS, et al. Early precursors and molecular determinants of tissue-resident memory CD ${ }^{+} \mathrm{T}$ lymphocytes revealed by single-cell RNA sequencing. Sci Immunol. 2020;5(47):eaaz6894.

21. Wang Z, et al. PD-1(hi) CD8(+) resident memory T cells balance immunity and fibrotic sequelae. Sci Immunol. 2019;4(36):eaaw1217.

22. Kinter AL, et al. The common gamma-chain cytokines IL-2, IL-7, IL-15, and IL-21 induce the expression of programmed death-1 and its ligands. J Immunol. 2008;181(10):6738-6746.

23. Hombrink P, et al. Erratum: programs for the persistence, vigilance and control of human CD8 $8^{+}$lung-resident memory T cells Nat Immunol. 2017;18(2):246.

24. Hombrink P, et al. Programs for the persistence, vigilance and control of human CD8(+) lung-resident memory T cells. Nat Immunol 2016;17(12):1467-1478.

25. Mackay LK, et al. The developmental pathway for CD103(+)CD8+ tissue-resident memory T cells of skin. Nat Immunol. 2013;14(12):1294-1301.

26. Mackay LK, et al. T-box transcription factors combine with the cytokines TGF- $\beta$ and IL-15 to control tissue-resident memory T cell fate. Immunity. 2015;43(6):1101-1111.

27. Freeman BE, et al. Regulation of innate CD8+ T-cell activation mediated by cytokines. Proc Natl Acad Sci U S A 2012;109(25):9971-9976.

28. Liu K, et al. IL-15 mimics T cell receptor crosslinking in the induction of cellular proliferation, gene expression, and cytotoxicity in CD8+ memory T cells. Proc Natl Acad Sci U S A. 2002;99(9):6192-6197.

29. Smeltz RB. Profound enhancement of the IL-12/IL-18 pathway of IFN-gamma secretion in human CD8+ memory T cell subsets via IL-15. J Immunol. 2007;178(8):4786-4792.

30. Martin MD, et al. Bystander responses impact accurate detection of murine and human antigen-specific CD8 T cells. J Clin Invest. 2019;129(9):3894-3908.

31. Utzschneider DT, et al. T cell factor 1-expressing memory-like CD8(+) T cells sustain the immune response to chronic viral infections. Immunity. 2016;45(2):415-427.

32. Hudson WH, et al. Proliferating transitory T cells with an effector-like transcriptional signature emerge from $\mathrm{PD}-1^{+}$stem-like 
CD8 ${ }^{+} \mathrm{T}$ cells during chronic infection. Immunity. 2019;51(6):1043-1058.

33. Yu S, et al. The TCF-1 and LEF-1 transcription factors have cooperative and opposing roles in T cell development and malignancy. Immunity. 2012;37(5):813-826.

34. Zhou X, et al. Differentiation and persistence of memory CD8(+) T cells depend on T cell factor 1. Immunity. 2010;33(2):229-240.

35. Maurice NJ, et al. The ugly duckling turned to swan: a change in perception of bystander-activated memory CD8 T Cells. J Immunol. 2021;206(3):455-462.

36. Butts CL, et al. Progesterone inhibits mature rat dendritic cells in a receptor-mediated fashion. Int Immunol. 2007;19(3):287-296.

37. Valenzuela $\mathrm{J}$, et al. The roles of IL-12 in providing a third signal for clonal expansion of naive CD8 T cells. J Immunol. 2002;169(12):6842-6849.

38. Kieper WC, et al. Il-12 enhances CD8 T cell homeostatic expansion. J Immunol. 2001;166(9):5515-5521.

39. Jameson SC, Masopust D. Understanding subset diversity in T cell memory. Immunity. 2018;48(2):214-226.

40. Duraiswamy J, et al. Phenotype, function, and gene expression profiles of programmed death-1(hi) CD8 T cells in healthy human adults. J Immunol. 2011;186(7):4200-4212.

41. Beura LK, et al. Normalizing the environment recapitulates adult human immune traits in laboratory mice. Nature. 2016;532(7600):512-516.

42. Sallusto F, et al. Two subsets of memory T lymphocytes with distinct homing potentials and effector functions. Nature. 1999;401(6754):708-712.

43. Kjer-Nielsen L, et al. MR1 presents microbial vitamin B metabolites to MAIT cells. Nature. 2012;491(7426):717-723.

44. Berkson JD, Prlic M. The MAIT conundrum — how human MAIT cells distinguish bacterial colonization from infection in mucosal barrier tissues. Immunol Lett. 2017;192:7-11.

45. Slichter CK, et al. Distinct activation thresholds of human conventional and innate-like memory T cells. JCI Insight. 2016;1(8):e86292

46. Voillet V, et al. Human MAIT cells exit peripheral tissues and recirculate via lymph in steady state conditions. JCI Insight. 2018;3(7):e98487.

47. Reantragoon R, et al. Antigen-loaded MR1 tetramers define T cell receptor heterogeneity in mucosal-associated invariant T cells. JExp Med. 2013;210(11):2305-2320.

48. Correction for Seo, et al. TOX and TOX2 transcription factors cooperate with NR4A transcription factors to impose CD8(+) T cell exhaustion. Proc Natl Acad Sci U S A. 2019;116(39):19761.

49. Kim CG, et al. VEGF-A drives TOX-dependent T cell exhaustion in anti-PD-1-resistant microsatellite stable colorectal cancers. Sci Immunol. 2019;4(41):eaay0555.

50. Blank CU, et al. Defining 'T cell exhaustion'. Nat Rev Immunol. 2019;19(11):665-674

51. Heim K, et al. TOX defines the degree of CD8+ T cell dysfunction in distinct phases of chronic HBV infection [published ahead of print]. October 23, 2020. Gut. https://doi.org/10.1136/gutjnl-2020-322404.

52. Guo L, et al. TOX correlates with prognosis, immune infiltration, and T cells exhaustion in lung adenocarcinoma. Cancer Med. 2020;9(18):6694-6709.

53. Kumar BV, et al. Human tissue-resident memory $\mathrm{T}$ cells are defined by core transcriptional and functional signatures in lymphoid and mucosal sites. Cell Rep. 2017;20(12):2921-2934.

54. Wang $\mathrm{X}$, et al. TOX promotes the exhaustion of antitumor $\mathrm{CD} 8^{+} \mathrm{T}$ cells by preventing PD1 degradation in hepatocellular carcinoma. J Hepatol. 2019;71(4):731-741

55. Arora M, et al. Expression pattern, regulation, and clinical significance of TOX in breast cancer. Cancer Immunol Immunother. 2021;70(2):349-363

56. Tucker CG, et al. Adoptive T cell therapy with IL-12-preconditioned low-avidity T cells prevents exhaustion and results in enhanced T cell activation, enhanced tumor clearance, and decreased risk for autoimmunity. J Immunol. 2020;205(5):1449-1460.

57. Page N, et al. Expression of the DNA-binding factor TOX promotes the encephalitogenic potential of microbe-induced autoreactive CD8(+) T cells. Immunity. 2018;48(5):937-950. 